\title{
Validating advanced wavefront control techniques on the SCExAO testbed/instrument
}

Guyon, Olivier, Lozi, Julien, Vievard, Sebastien, Belikov, Ruslan, Bendek, Eduardo, et al.

Olivier Guyon, Julien Lozi, Sebastien Vievard, Ruslan Belikov, Eduardo Bendek, Steven Bos, Thayne Currie, Vincent Deo, Michael Fitzgerald, Damien Gratadour, Tyler Groff, Nemanja Jovanovic, Hajime Kawahara, Takayuki Kotani, Tomoyuki Kudo, Coline Lopez, Hatem Ltaief, Jared Males, Frantz Martinache, Marc-Antoine Martinod, Benjamin A. Mazin, Kelsey Miller, Barnaby Norris, Mamadou Ndiaye, Eugene Pluzhnyk, Ananya Sahoo, Arnaud Sevin, Nour Skaf, Frans Snik, Motohide Tamura, Alison Wong, "Validating advanced wavefront control techniques on the SCEXAO testbed/instrument," Proc. SPIE 11448, Adaptive Optics Systems VII, 114481Z (13 December 2020); doi: $10.1117 / 12.2562723$ 


\title{
Validating advanced wavefront control techniques on the SCExAO testbed/instrument*
}

Olivier Guyon ${ }^{\mathrm{a}, \mathrm{b}, \mathrm{c}, \mathrm{d}}$, Julien Lozi ${ }^{\mathrm{a}}$, Sebastien Vievard ${ }^{\mathrm{a}, \mathrm{d}}$, Ruslan Belikov ${ }^{\mathrm{e}}$, Eduardo Bendek ${ }^{\mathrm{f}}$, Steven Bos ${ }^{\mathrm{g}}$, Thayne Currie ${ }^{\mathrm{a}, \mathrm{e}}$, Vincent Deo ${ }^{\mathrm{a}, \mathrm{h}}$, Michael Fitzgerald ${ }^{\mathrm{i}}$, Damien Gratadour ${ }^{\mathrm{h}, \mathrm{j}}$, Tyler Groff ${ }^{k}$, Nemanja Jovanovic ${ }^{1}$, Hajime Kawahara ${ }^{\mathrm{m}}$, Takayuki Kotani ${ }^{\mathrm{d}}$, Tomoyuki Kudo ${ }^{\mathrm{a}}$, Coline Lopez ${ }^{\mathrm{n}}$, Hatem Ltaief ${ }^{\mathrm{o}}$, Jared Males ${ }^{\mathrm{b}}$, Frantz Martinache ${ }^{\mathrm{n}}$, Marc-Antoine Martinod ${ }^{\mathrm{q}}$, Benjamin A. Mazin ${ }^{\mathrm{p}}$, Kelsey Miller ${ }^{\mathrm{g}}$, Barnaby Norris ${ }^{\mathrm{q}}$, Mamadou N'Diaye ${ }^{\mathrm{n}}$, Eugene Pluzhnyk ${ }^{\mathrm{e}}$, Ananya Sahoo ${ }^{\mathrm{a}}$, Arnaud Sevin ${ }^{\mathrm{h}}$, Nour Skaf ${ }^{\mathrm{a}}$, Frantz Snik ${ }^{\mathrm{g}}$, Motohide Tamura ${ }^{\mathrm{m}, \mathrm{d}}$, and Alison Wong $^{\mathrm{q}}$

${ }^{a}$ Subaru Telescope, National Astronomical Observatory of Japan, National Institutes of Natural Sciences (NINS), 650 North A'ohōkū Place, Hilo, HI, 96720, U.S.A.

${ }^{\mathrm{b}}$ Steward Observatory, University of Arizona, Tucson, AZ, U.S.A. ${ }^{\mathrm{c}}$ College of Optical Sciences, University of Arizona, Tucson, AZ, U.S.A.

${ }^{\mathrm{d} A s t r o b i o l o g y}$ Center of NINS, 2-21-1, Osawa, Mitaka, Tokyo, Japan eNASA-Ames Research Center, Moffett Blvd., Moffett Field, CA, U.S.A.

${ }^{\mathrm{f} J}$ Jet Propulsion Laboratory, California Institute of Technology, 4800 Oak Grove Dr.,Pasadena, CA 91109, U.S.A.

'Leiden Observatory, Leiden University, PO Box 9513, 2300 RA Leiden, The Netherlands

${ }^{\text {h} L E S I A, ~ O b s e r v a t o i r e ~ d e ~ P a r i s, ~ U n i v e r s i t e ́ ~ P S L, ~ C N R S, ~ S o r b o n n e ~ U n i v e r s i t e ́, ~ U n i v e r s i t e ́ ~ d e ~}$ Paris, 5 place Jules Janssen, 92195 Meudon, France

${ }^{i}$ Department of Physics \& Astronomy, 430 Portola Plaza, University of California, Los Angeles, CA 90095, U.S.A.

${ }^{j}$ Research School of Astronomy and Astrophysics, Australian National University, Canberra,

ACT 2611, Australia

${ }^{k}$ NASA-Goddard Space Flight Center, Greenbelt, MD, USA

${ }^{1}$ California Institute of Technology, 1200 E California Blvd, Pasadena, CA 91125, U.S.A.

${ }^{\mathrm{m}}$ The University of Tokyo, 7-3-1, Hongo, Bunkyo-ku, Tokyo 113-0033, Japan;

${ }^{n}$ Université Côte d'Azur, Observatoire de la Côte d'Azur, CNRS, Laboratoire Lagrange, France.

${ }^{\circ}$ Extreme Computing Research Center, King Abdullah University of Science and Technology, Thuwal, Saudi Arabia

${ }^{\mathrm{P}}$ Department of Physics, University of California, Santa Barbara, 93106, U.S.A.

" Sydney Institute for Astronomy, School of Physics, The University of Sydney, NSW 2006, Australia

\begin{abstract}
The Subaru Coronagraphic Extreme Adaptive Optics (SCExAO) serves both a science instrument in operation, and a prototyping platform for integrating and validating advanced wavefront control techniques. It provides a modular hardware and software environment optimized for flexible prototyping, reducing the time from concept
\end{abstract}

${ }^{*}$ Based on data collected at Subaru Telescope, which is operated by the National Astronomical Observatory of Japan.

Further author information: (Send correspondence to O.G.)

O.G.: E-mail: guyon@naoj.org, Telephone: 18182938826

Adaptive Optics Systems VII, edited by Laura Schreiber, Dirk Schmidt, Elise Vernet, Proc. of SPIE Vol. 11448, 114481Z · C 2020 SPIE · CCC code: 0277-786X/20/\$21 · doi: 10.1117/12.2562723 
formulation to on-sky operation and validation. This approach also enables external research group to deploy and test new hardware and algorithms.

The hardware architecture allows for multiple subsystems to run concurrently, sharing starlight by means of dichroics. The multiplexing lends itself to running parallel experiments simultaneously, and developing sensor fusion approaches for increased wavefront sensing sensitivity and reliability. Thanks to a modular realtime control software architecture designed around the CACAO package, users can deploy WFS/C routines with full low-latency access to all cameras data streams. Algorithms can easily be shared with other cacao-based AO systems at Magellan (MagAO-X) and Keck.

We highlight recent achievements and ongoing activities that are particularly relevant to the development of high contrast imaging instruments for future large ground-based telescopes (ELT, TMT, GMT) and space telescopes (HabEx, LUVOIR). These include predictive control and sensor fusion, PSF reconstruction from AO telemetry, integrated coronagraph/WFS development, focal plane speckle control with photon counting MKIDS camera, and fiber interferometry.

We also describe upcoming upgrades to the WFS/C architecture: a new 64x64 actuator first stage DM, deployment of a beam switcher for concurrent operation of SCExAO with other science instruments, and the ULTIMATE upgrade including deployment of multiple LGS WFSs and an adaptive secondary mirror.

Keywords: Adaptive Optics, High Contrast Imaging, Wavefront Sensing, Atmospheric Turbulence

\section{INTRODUCTION}

High contrast imaging systems on large space and ground telescopes enable direct imaging and spectroscopic characterization of exoplanets and circumstellar disks. ${ }^{1-6}$ Large ground-based telescopes are particularly well suited for near-IR thermal imaging of young massive planets, ${ }^{7-9}$ while the deeper contrast levels accessible from space provide access to starlight reflected off giant planets and circumstellar disks. ${ }^{10-12}$ From both ground and space, direct imaging and spectrocospic analysis of potentially habitable planets should become possible with future planned telescopes, but remains a significant technical challenge. ${ }^{13-15}$

While starlight suppression systems have achieved deep raw contrast in vacuum testing, high contrast imaging perfromance will be limited by the ability to accurately and efficiently measure residual wavefront errors. High perfrormance Wavefront sensing (WFS) is essential to both achieve deep raw contrast by way of wavefront control, and to calibrate residual light in the science image to reliably recover faint companions, ideally to the photon noise limit. Accurate and efficent WFS is thus essential to high contrast imaging instruments and missions, both ground and space-based.

On ground-based telescopes, rapidly evolving atmospheric turbulence can be mitigated by extreme adaptive optics (XAO) correction. ${ }^{16}$ Current XAO systems can deliver up to $10^{-4}$ raw contrast on typical bright targets at angular separations of a few $\lambda / D .{ }^{17}$ The main fundamental limits to this raw contrast level are :

1. Temporal lag. Fast, low-latency correction is required to keep up with continuously evolving atmospheric turbulence. Allowable latency scales as the $6 / 5$-th power of required wavefront error, so XAO systems are particularly demanding.

2. WFS sensitivity to photon noise. XAO systems require high precision wavefront measurements over a large number of degrees of freedom at high speed. Even on bright stars, photon noise is therefore a major source of error.

3. Differential chromatic effects between WFS and science image due to the wavelength dependence in refractive index of air, and due to diffraction propagation between atmospheric layers.

4. Amplitude (scintillation) created by diffraction propagation through turbulence. 
Powerful point-spread function (PSF) subtraction techniques ${ }^{18,19}$ used in conjunction with angular and spectral differential imaging (ADI, SDI) ${ }^{20,21}$ can yield contrast levels $\approx 10-100 \times$ deeper than raw contrast. ${ }^{22}$ But this level of suppression is still falls well short of the photon noise limit. Unknown residual wavefront errors creating speckle noise in the focal plane limit detection robustness and detection contrast limit, as there is ambiguity between speckles and actual astrophysical sources.

Space-based HCI systems suffer from the same fundamental limits, but at a different scale. Without atmospheric turbulence, wavefront errors are smaller and slower, allowing deeper raw contrast. The XAO correction control loop operates slower, gathering more starlight for each measurement so that WFS photon noise can be reduced to the required contrast level. Measurements are primarly performed in the focal plane to avoid non-common path errors, and low-order aberrations can be tracked with a faster sensor making use of bright starlight rejected by the coronagraph. Ultimately, contrast depth and detection limit are also limited by WFS accuracy and sensitivity, as picometer-level wavefront variations can create speckles with brightness comparable to a habitable planet orbiting a Sun-like star.

The Subaru Coronagraphic Extreme Adaptive Optics (SCExAO) is both a science instrument and a prototyping platform for integrating and validating XAO wavefront control techniques. We describe in $\S 2$ its hardware configuration and in $\S 3$ how it provides an environment suitable for flexible prototyping, reducing the time from concept formulation to on-sky operation and validation. Main current research themes supported by SCExAO are described in $\S 4$ along with specific examples.

\section{HARDWARE CONFIGURATION}

The SCExAO instrument is installed on the Nasmyth platform of the $8.2 \mathrm{~m}$ Subaru Telescope. The instrument platform spans $\approx 8 \times 7 m$, and hosts the facility AO system as well as SCExAO and its instrument modules. Light from the telescope is first corrected by the AO facility system, then sent to SCExAO for additional correction by a 2000-actuator MEMS type deformable mirror.
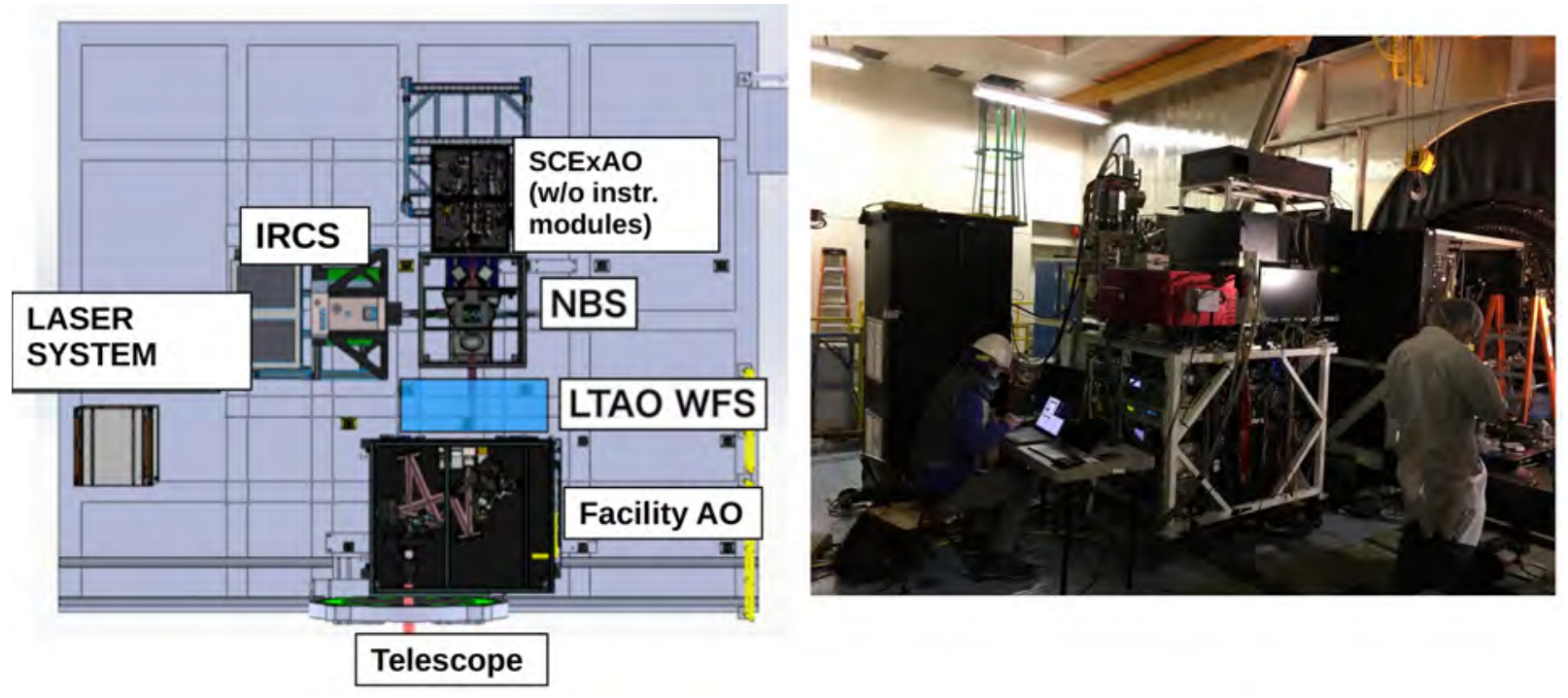

Figure 1. (left) Instrument layout on Subaru's Nasmyth infrared platform. The layout shows the configuration after deployment of the Nasmyth beam switcher (NBS) currently in development. (right) Image of the SCExAO instrument on the platform.

\subsection{Nasmyth Platform Layout}

The facility AO system, currently a 188-element curvature system, will be upgraded in 2021 to a 3200-element deformable mirror and a near-IR pyramid WFS. Wavefront sensing can be provided by visible or near-IR starlight, or by four LGS sensors for laser tomography. 
The facility AO system can feed multiple instruments, including SCExAO and the Infrared Camera and Spectrograph (IRCS). The overhead crane is currently (2020) used to physically move instruments in the AO focus. A Nasmyth beam switcher (NBS) currently under development will provide crane-free beam switching, and will enable modes of operation where multiple instruments can operate simultaneously with the appropriate dichroic splitter setting. Figure 1 (left) shows the final configuration with the NBS.
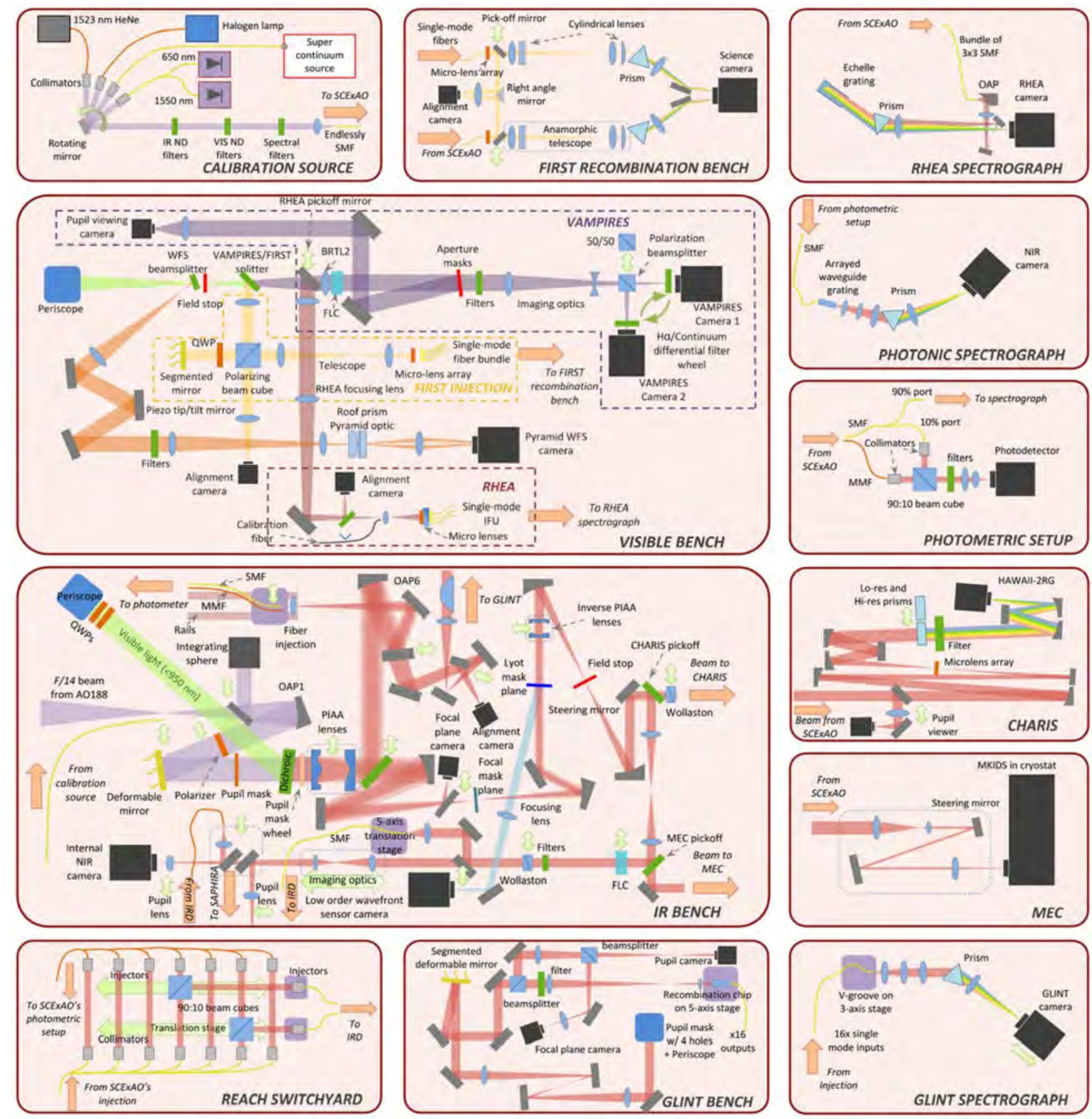

Figure 2. SCExAO instrument optical train showing the two main optical bench and instrument modules.

\subsection{SCExAO System Overview}

The SCExAO system, shown in Fig. 1 (right), delivers fine wavefront correction and starlight suppression for mutliple instrument modules. Current (2020) SCExAO instrument modules are routinely available for both 
on-sky and off-sky operation :

- CHARIS,$^{23}$ a near-IR integral field spectrograph operating from 1.1 to $2.4 \mu m$, which is the main workhorse high-contrast science instrument used with SCExAO, achieving contrasts of $10^{-6}$ at $0^{\prime \prime} 5^{24}$ (see paper 11448-330 of this meeting). SCExAO/CHARIS has yielded new discoveries of substellar companions, spectral characterization of known exoplanets and other objects, and multi-wavelength spatial characterization of planet-forming disks. ${ }^{25-30}$

- VAMPIRES ${ }^{31}$, a dual-beam visible light imager, with polarization and spectral differential imaging modes, and aperture masking. SCExAO/VAMPIRES is used to resolve circumstellar material at small angular separations and can detect accreting protoplanets in $H_{\alpha} \cdot{ }^{32}$

- FIRST, a visible light spectro-interferometer. ${ }^{33}$

- GLINT, a near-IR photonic nuller. ${ }^{31,34}$

- MEC $^{35}$, the MKID exoplanet camera, a wavelength-resolving noise-free photon counting imaging camera. MEC typically operates in parallel with CHARIS taking shorter wavelength light.

- REACH ${ }^{36}$ which injects planet light into a single mode fiber for high resolution $(\mathrm{R} \approx 100,000)$ near-IR spectroscopy with the existing IRD spectrograph.

- NIR-PDI ${ }^{37}$ uses a high frame rate near-IR camera for polarization differential imaging

- RHEA, ${ }^{38}$ a narrow-field visible high resolution integral field spectrograph

Additional experimental hardware can be deployed for specific tests.

Fig. 2 shows a block diagram of SCExAO including the above modules.

\section{ACCESS AND OPERATION AS A TEST PLATFORM}

\subsection{Testbed capabilities}

The SCExAO instrument platform has been optimized to facilitate off-sky testing to support development and validation of new WFS concepts prior to on-sky deployment. The instrument is continuously available (day and night) for off-sky testing outside of the pre-scheduled on-sky operation nights (typically $\approx 16$ nights per yr).

Off-sky testing uses an internal calibration source (top left, Fig. 2) capable of simultaneously feeding optical and near-IR broadband light with selectable independent brightness settings. Multiple high cameras and sensors are available for WFS development, as listed in table 1. Most of these can run simultaneously thanks to selectable dichroic splitters, so that source light can be split in wavelength of flux according to the goals of the experiement.

Table 1. Wavefront sensors and high speed cameras.

\begin{tabular}{|l|c|c|c|c|}
\hline Sensor & Wavelength & Speed & Type & Status \\
\hline Curvature APD array & VIS & $2 \mathrm{kHz}$ & 188 APDs & in operation \\
\hline LTAO SHWFS x5 & VIS & $\mathrm{kHz}$ & $25 \times 25$ cells, sCMOS & 2021 \\
\hline VIS Pyramid & $600 \mathrm{~nm}-900 \mathrm{~nm}$ & $3.5 \mathrm{kHz}$ & $120 \times 120$ EMCCD & in operation \\
\hline LOWFS & $0.9-1.7 \mu \mathrm{m}$ & $<6 \mathrm{kHz}$ & InGaAs array & in operation \\
\hline MKID Exoplanet Camera & $0.9-1.4 \mu \mathrm{m}$ & $2 \mathrm{kHz}$ & MKID photon counting & in operation \\
\hline VAMPIRES (FPWFS) x2 & $0.6-0.8 \mu \mathrm{m}$ & $1 \mathrm{kHz}$ & $512 \times 512$ EMCCD & in operation \\
\hline NIR Pyramid & $0.9-1.8 \mu \mathrm{m}$ & $3 \mathrm{kHz}$ & MCT array & 2021 \\
\hline GLINT interferometer & $0.9-1.7 \mu \mathrm{m}$ & $1.4 \mathrm{kHz}$ & InGaAs & in operation \\
\hline
\end{tabular}



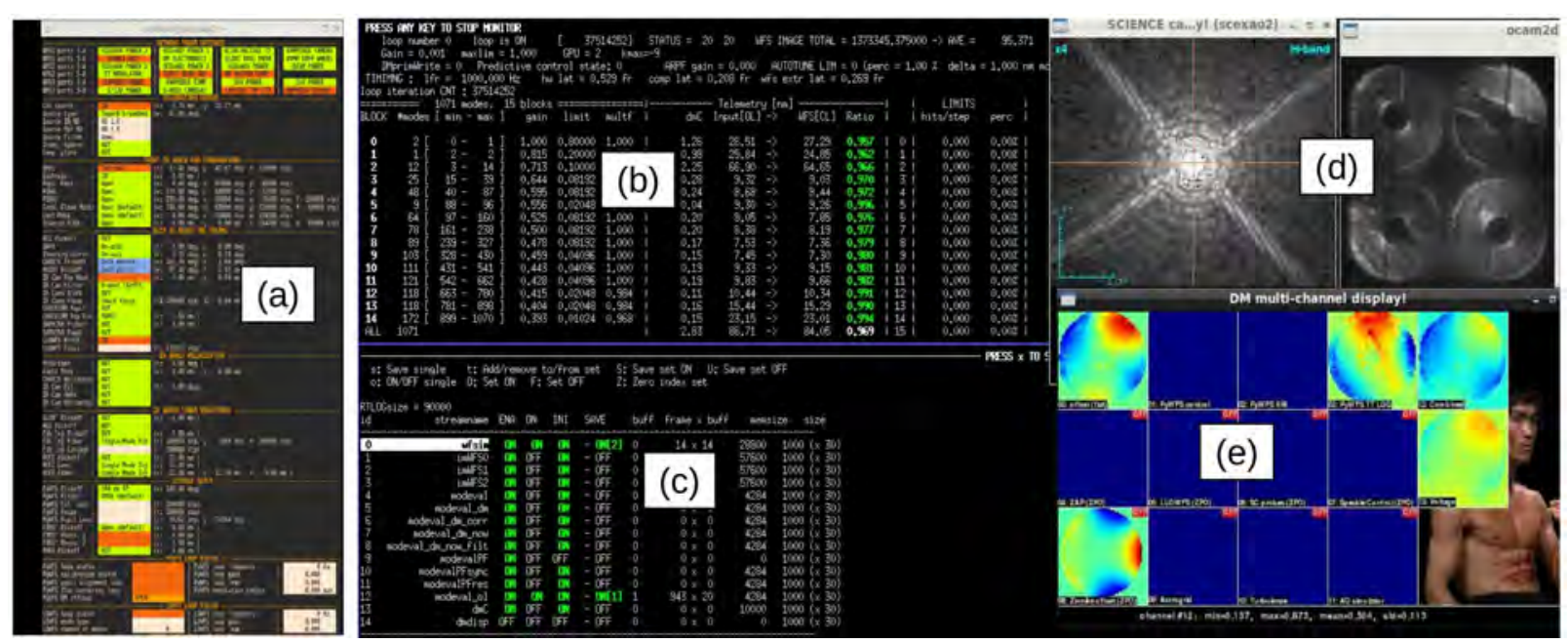

Figure 3. SCExAO control screens. (a) Status of control stages, filter wheels and other opto-mechanical devices. (b) Summary of real-time modal telemetry for the main AO control loop. (c) Telemetry storage to disk control screen. (d) Viewers for real-time image streams: focal plane image (left) and pyramid WFS camera (right). (e) Deformable Mirror (DM) control channels.

\subsection{Access and User interface}

Remote access to the instrument is provided by remote login to the instrument control and real-time control computers. An example screenshot of the instrument control screen is shown in Fig. 3.

Scheduling for tests is coordinated with a shared calendar, and live interaction with the support team is done through the Slack communication platform.

\subsection{Software}

To aid WFS development activities, the instrument software supports saving all telemetry to disk and provides uniform software interfaces for deployment of algorithms. Software frameworks and data formats are based on the open-source milk and cacao packages which are also used for the MagAO-X system at the Magellan telescope, and inter-compatible with the COMPASS simulation framework.
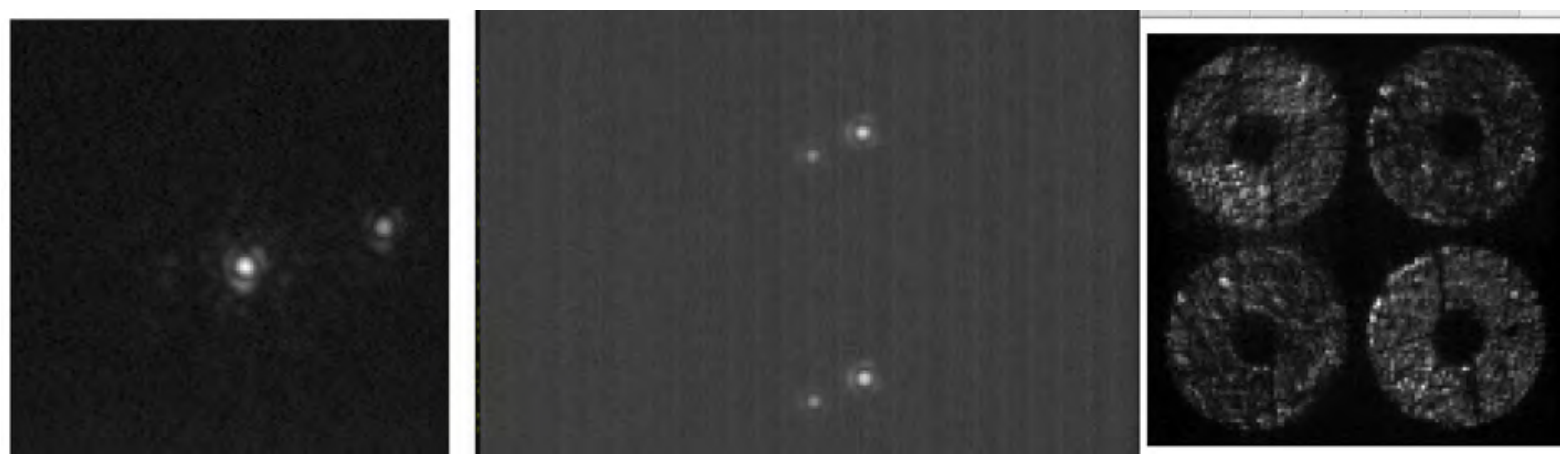

Figure 4. Example telemetry. Simultaneous on-sky frames from VAMPIRES cam1 at 675nm (left, single frame from 500 $\mathrm{Hz}$ stream), Near-IR scicam at 1630nm (center, single frame from $1 \mathrm{kHz}$ stream) in PDI mode, and visible Pyramid WFS (right, single frame from $2 \mathrm{kHz}$ stream). Data acquired on $\sigma$ Ori double star, 0.25 arcsec separation, on Aug 8, 2020.

\subsubsection{Telemetry}

The WFS cameras listed in table 1 are made available for real-time use as streams in shared memory. A common format, using the ImageStreamIO format is adopted, providing read/write access through $\mathrm{C}$ and Python 
languages APIs. Streams can be saved to disk for later inspection, as illustrated in Fig. 4. Sub-frame synchronization between streams at the $\approx 20 \mu \mathrm{s}$ level is achieved by accurate measurement of each stream latency, using poking on the system DM for common time reference. A common TCP-based stream transfer routine is used to move real-time streams originating from separate computers to the same physical computer for realtime processing, as required. Dedicated 100GBE and 10GBE fiber links between computers provide low-latency connectivity.

\subsubsection{Algorithm Deployment}

Several options are supported for algorithm deployment for WFS development. Low latency real-time code can be deployed as compiled $\mathrm{C}$ or $\mathrm{C}++$ code using the telemetry stream API. Python code can also be deployed for less performance-critical code. Finally, raw WFS frames and DM commands can be exchanged by file system or network independently of the stream API if the associated latency is acceptable.

The SCExAO real-time control software, provided by the compute and control for adaptive optics (CACAO) package ${ }^{39}$ provides high-level functions that are particularly useful for WFS algorithms deployment:

- Deformable mirror (DM) multi-channel control - The system DM is exposed to the users not as a single actuator array, but as twelve (12) independent DM channels. Low-latency software automatically adds all channels whenever one of the channels is updated, and send to the physical DM the sum of all 12 channels. It thus appears to users as if the system has $12 \mathrm{DMs}$, and a specific experiment can take control of one of these channels without requiring coordination with other processes writing to the DM.

- Automatic zero point offload between control loops - Individual DM channels can be configured to be offload channels, where each update to the channel will both issue a command to the DM and change the convergence point (zero point) of the primary AO control loop. This is necessary for WFS algorithms requiring DM probing, so that the probes are not erased by the primary AO control loop.

\section{AREAS OF ACTIVE RESEARCH}

Hardware and software capabilities described in $\S 2$ and 3 are supporting multiple WFS research activies, which fall under four main themes: Wavefront control algorithms, Focal plane sensing and referencing, and high efficiency wavefront sensing.

\subsection{Wavefront control algorithms (making optimal use of available WFS information)}

Wavefront control systems operate in a control loop consisting of three steps: (1) wavefont sensor data is acquired; (2) wavefront is estimated from measurements, and (3) the new estimate is converted to a correction which is incrementally applied to the corrective elements (such as deformable mirror).

Advances in wavefront control algorithms are aimed at better using available WFS information to improve wavefront correction and calibration.

Research questions under this research theme include :

- Predictive control: Can the temporal wavefront evolution be leveraged to improve wavefront correction? Wavefront errors exhibit strong spatial and temporal correlations due to wind-driven turbulence (ground telescopes), thermal drifts or vibrations (space and ground telescopes).

- Non-linear WF estimation: Can additional wavefront information be extracted from nonlinear reconstruction? Current WFS schemes assume linearity between pixel intensity and wavefront phase (pupil plane sensors), or between deformable mirror displacement and focal plane complex amplitude (focal plane sensors). Including non-linear effects can increase wavefront estimate accuracy over a wider range of conditions.

- Dynamical self-calibration: Can the wavefront reconstruction algorithm optimally learn and adapt? Most wavefront control system rely on pre-computed or pre-measured calibration of WFS response to deformable mirror commands. This response does evolve with time, and is itself a function of the wavefront state. Wavefront sensor telemetry contains valuable information to derive or constrain the system response. 

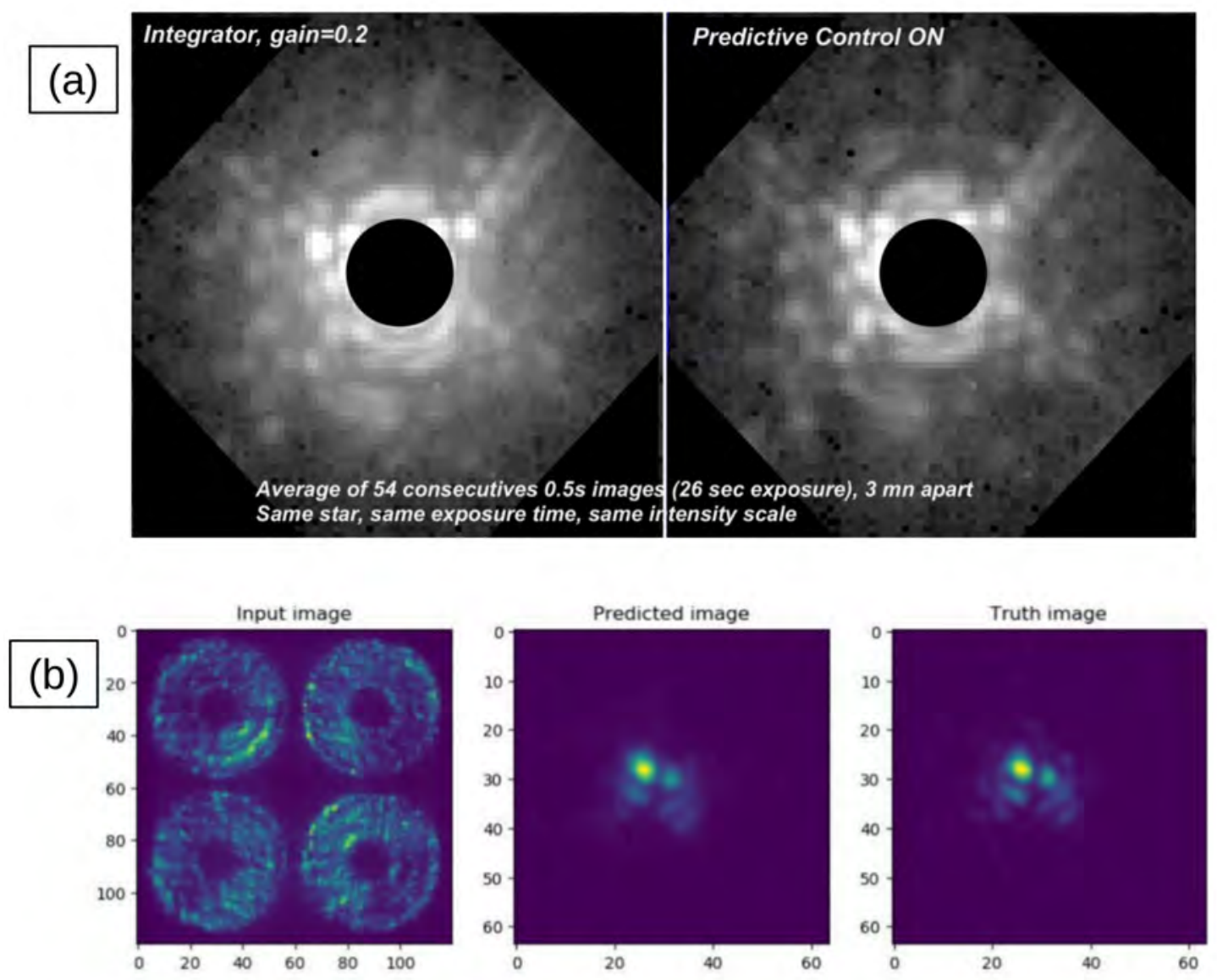

Figure 5. Advanced wavefront control algorithms - selected examples. (a) On-sky demonstration of predictive control using the EOF framework, yielding a 3x contrast gain. (b) Evidence of non-linear wavefront sensing information recovery using a neural network. See text for details.

\subsection{Focal plane wavefront sensing and referencing}

Focal plane images, including the post-coronagraphic science image, are uniquely powerful for high contrast imaging wavefront sensing and control, offering both sensitivity and immunity from non-common path aberrations.

Such images can be used for wavefront sensing, either as the primary source of wavefront estimation, or as a reference for fast control loops using pupil-plane wavefront sensors. SCExAO provides multiple high speed low noise focal plane sensors in visible and nearIR. They are made available for real-time processing to support this research theme.

Research questions under this theme include:

- Speckle control: How can Focal plane speckle probing nulling be optimized for speed, sensitivity and robustness? In speckle nulling, DM probes are added to perturb the focal plane image. This perturbation is measured to extract wavefront information as complex amplitude of the focal plane speckles, and can then be employed to null speckles (Fig. 6 (a)) or optimize PSF quality (Fig. 6 (b)). Deployment of efficient and robust real-time focal plane wavefront control for ground-based systems remains a significant algorithmic challenge.

- Linear dark field control ${ }^{40}$ (LDFC): Can bright speckles outside the high contrast be used to stabilize the wavefront state in a linear loop? LDFC utilizes the linear response of the uncorrected 

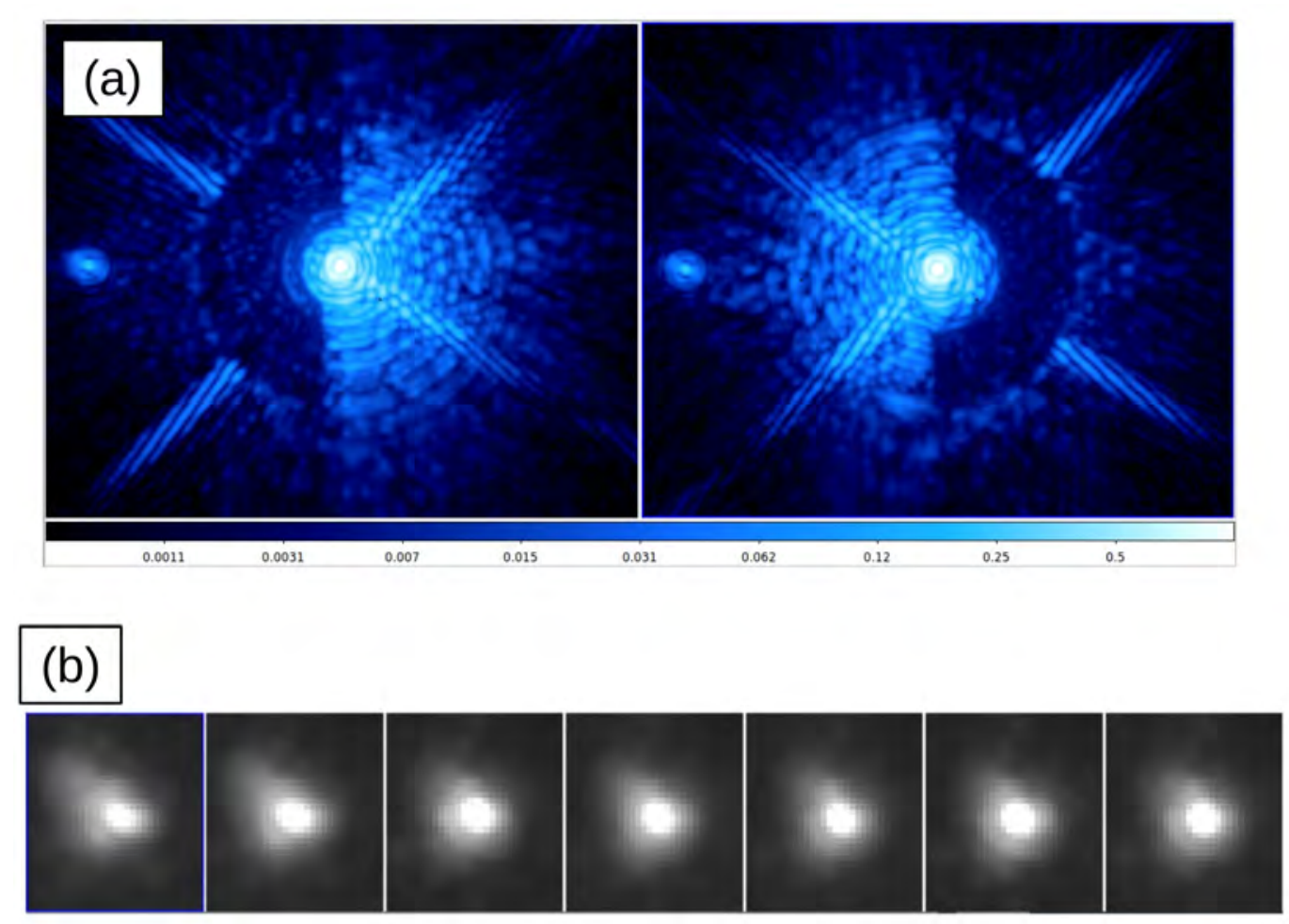

Figure 6. Focal plane wavefront control - selected examples. (a) Speckle nulling showing left and right side dark hole configurations (internal source). (b) On-sky demonstration of the DrWho focal plane image referencing algorithm. See text for details.

but photon-rich region in the focal plane (the "bright field"; BF) to wavefront perturbations that affect both the BF and the photon-starved DF. LDFC does not require DM probing to operate, resulting in a temporally correlated DF well suited for further suppression using PSF subtraction techniques. LDFC has been tested in the laboratory at contrasts necessary to image planets in reflected light; ${ }^{41}$ tests using the SCExAO internal source and on sky thus far are encouraging (Miller et al. submitted; Bos et al. in prep.).

- Multi-star wavefront control: Can focal-plane wavefront contol be applied to double stars? Many targets of interest are double star, requiring focal plane wavefront control to be tailored. ${ }^{42}$

- Coherent differential imaging: Can coherence be measured and can it separate speckles from planets? Speckle modulation induced either by intentional probing or fast residual atmospheric turbulence can be used to measure both the complex amplitude of speckles and their coherence with startlight. The incoherent residual contains the planet light.

\subsection{High efficiency wavefront sensing}

Optimal use of the fixed available amount of starlight is essential to mitigate the photon noise WFS error.

Research questions under this theme include:

- New optical concepts for WFS - Development of high efficiency WFS concepts is essential to high contrast imaging. Photonics technologies, and in particular on-chip waveguides and coherent couplers, offer new possibilities to optimize WFS efficiency.

- Can measurements from multiple sensors be combined? Sensor fusion to combine measurements across a wide wavelenght range can increase sensitivity. This is especially relevant to post-coronagraphic 


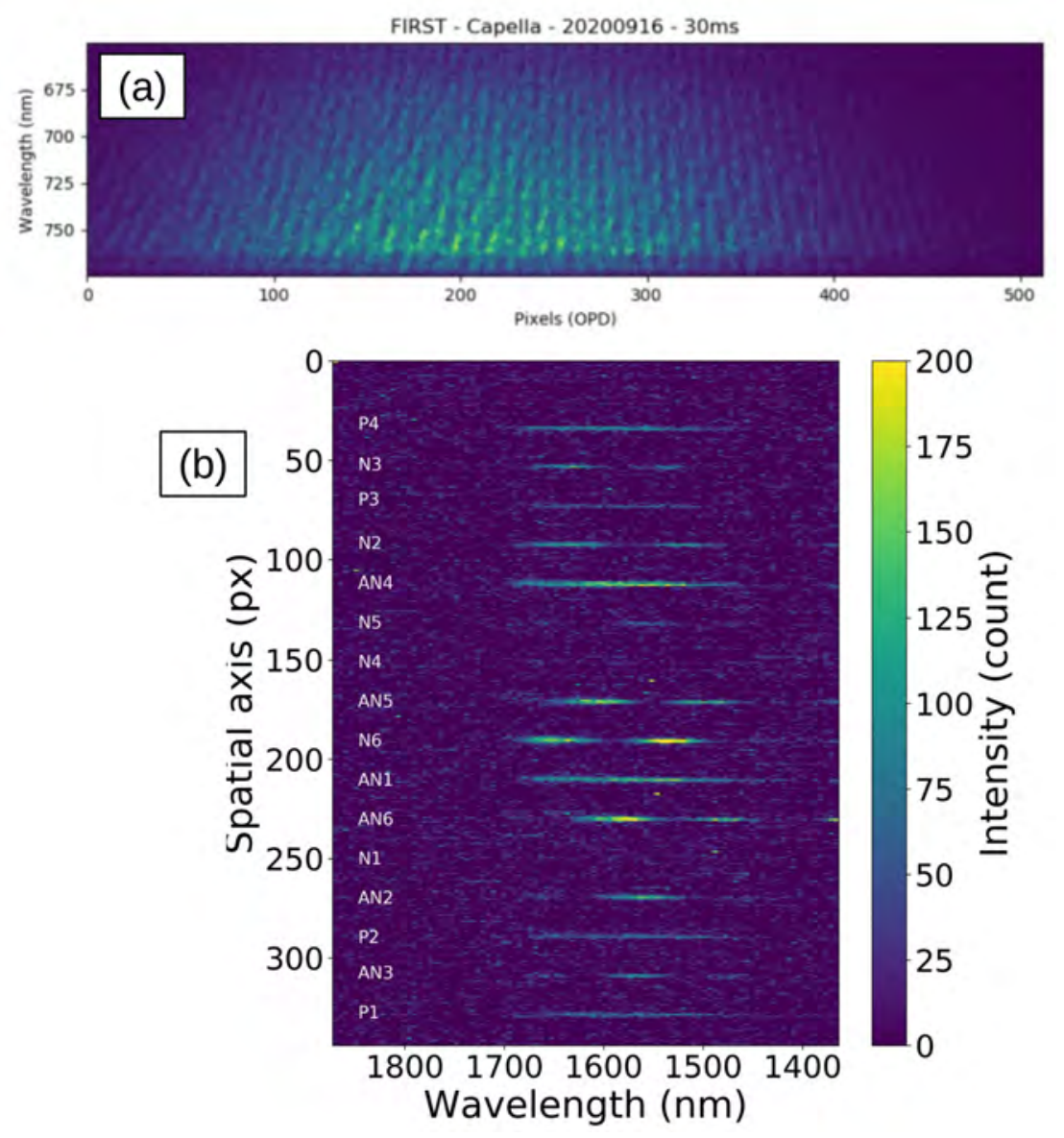

Figure 7. Interferometric WFS - selected examples. (a) On-sky image of of the Capella double star acquired with the FIRST instrument module. (b) On-sky image of Arcturus acquired with the GLINT instrument module. See text for details.

focal plane wavefront sensing approaches that only use a narrow spectral bandwidth $(\approx 20 \%$, limited by the starlight suppression chromaticity).

SCExAO provides wavefront sensors that operate concurrently at several spectral bands to explore this topic. The FIRST and GLINT instruments, both interferometers, also encode wavefront information at high sensitivity with wavelength resolution, thanks to new photonics technologies (see Fig. 7).

\section{FUTURE WORK AND PERSPECTIVES}

The SCExAO system is well-suited for research and development of new WFS techniques thanks to its hardware configuration on the nearIR Nasmyth platform of the Subaru Telescope. Multiple research activities are supported to explore promising paths for improving wavefront correction and calibration. Most of the approaches under development are applicable to both ground and space high contrast imaging instruments and projects.

For ground-based HCI instruments, the combination of predictive control, multi-wavelength sensing, new optical sensing and smarter algorithms can potentially push performance significantly beyond the previously assumed $1 e 5$ raw contrast limit at small angular separations. ${ }^{43}$ High performance computing technologies are an essential component of these developments for ground-based telescopes, as control loops operate at and above $k H z$ frequency and handle large data bandwidth. 
Additionally, precise bias-free calibration of residual wavefront errors holds the potential to acquire images and spectra of exoplanets at the photon noise limit over long exposure times. This last goal would allow future large ground and space telescopes to study the atmospheres of habitable exoplanets at high accuracy to support the search for life outside our solar system. Advances in wavefront sensing techniques, including low-noise detectors, provide the ability for complete real-time measurement of the wavefront state, where all disturbances producing focal plane speckles are also contained in the collection of WFS telemetry streams, making it essentially impossible for unwanted speckles to hide from telemetry. Exploiting the full potential of WFS telemetry, while a significant computational challenge, is key to achieving the photon-noise calibration limit.

\section{ACKNOWLEDGMENTS}

The development of SCExAO was supported by the Japan Society for the Promotion of Science (Grant-in-Aid for Research \#23340051, \#26220704, \#23103002, \#19H00703 \& \#19H00695), the Astrobiology Center of the National Institutes of Natural Sciences, Japan, the Mt Cuba Foundation and the director's contingency fund at Subaru Telescope. The authors wish to recognize and acknowledge the very significant cultural role and reverence that the summit of Maunakea has always had within the Hawaiian community. We are very fortunate to have the opportunity to conduct observations from this mountain.

\section{REFERENCES}

[1] Marois, C., Macintosh, B., Barman, T., Zuckerman, B., Song, I., Patience, J., Lafrenière, D., and Doyon, R., "Direct Imaging of Multiple Planets Orbiting the Star HR 8799," Science 322, 1348- (Nov. 2008).

[2] Marois, C., Zuckerman, B., Konopacky, Q. M., Macintosh, B., and Barman, T., "Images of a fourth planet orbiting HR 8799," Nature 468, 1080-1083 (Dec 2010).

[3] Barman, T. S., Konopacky, Q. M., Macintosh, B., and Marois, C., "Simultaneous Detection of Water, Methane, and Carbon Monoxide in the Atmosphere of Exoplanet HR8799b," Astrophysical Journal 804, 61 (May 2015).

[4] Currie, T., Burrows, A., Itoh, Y., Matsumura, S., Fukagawa, M., Apai, D., Madhusudhan, N., Hinz, P. M., Rodigas, T. J., Kasper, M., Pyo, T.-S., and Ogino, S., "A Combined Subaru/VLT/MMT 1-5 $\mu \mathrm{m}$ Study of Planets Orbiting HR 8799: Implications for Atmospheric Properties, Masses, and Formation," Astrophysical Journal 729, 128 (Mar. 2011).

[5] Schneider, G., Grady, C. A., Hines, D. C., Stark, C. C., Debes, J. H., Carson, J., Kuchner, M. J., Perrin, M. D., Weinberger, A. J., Wisniewski, J. P., Silverstone, M. D., Jang-Condell, H., Henning, T., Woodgate, B. E., Serabyn, E., Moro-Martin, A., Tamura, M., Hinz, P. M., and Rodigas, T. J., "Probing for Exoplanets Hiding in Dusty Debris Disks: Disk Imaging, Characterization, and Exploration with HST/STIS Multi-roll Coronagraphy," Astronomical Journal 148, 59 (Oct. 2014).

[6] Esposito, T. M., Kalas, P., Fitzgerald, M. P., Millar-Blanchaer, M. A., Duchêne, G., Patience, J., Hom, J., Perrin, M. D., De Rosa, R. J., Chiang, E., Czekala, I., Macintosh, B., Graham, J. R., Ansdell, M., Arriaga, P., Bruzzone, S., Bulger, J., Chen, C. H., Cotten, T., Dong, R., Draper, Z. H., Follette, K. B., Hung, L.-W., Lopez, R., Matthews, B. C., Mazoyer, J., Metchev, S., Rameau, J., Ren, B., Rice, M., Song, I., Stahl, K., Wang, J., Wolff, S., Zuckerman, B., Ammons, S. M., Bailey, V. P., Barman, T., Chilcote, J., Doyon, R., Gerard, B. L., Goodsell, S. J., Greenbaum, A. Z., Hibon, P., Hinkley, S., Ingraham, P., Konopacky, Q., Maire, J., Marchis, F., Marley, M. S., Marois, C., Nielsen, E. L., Oppenheimer, R., Palmer, D., Poyneer, L., Pueyo, L., Rajan, A., Rantakyrö, F. T., Ruffio, J.-B., Savransky, D., Schneider, A. C., Sivaramakrishnan, A., Soummer, R., Thomas, S., and Ward-Duong, K., "Debris Disk Results from the Gemini Planet Imager Exoplanet Survey's Polarimetric Imaging Campaign," Astronomical Journal 160, 24 (July 2020).

[7] Currie, T., Cloutier, R., Brittain, S., Grady, C., Burrows, A., Muto, T., Kenyon, S. J., and Kuchner, M. J., "Resolving the HD 100546 Protoplanetary System with the Gemini Planet Imager: Evidence for Multiple Forming, Accreting Planets," Astrophysical Journal Letters 814, L27 (Dec 2015).

[8] Macintosh, B., Graham, J. R., Barman, T., De Rosa, R. J., Konopacky, Q., Marley, M. S., Marois, C., Nielsen, E. L., Pueyo, L., Rajan, A., Rameau, J., Saumon, D., Wang, J. J., Patience, J., Ammons, M., Arriaga, P., Artigau, E., Beckwith, S., Brewster, J., Bruzzone, S., Bulger, J., Burningham, B., Burrows, A. S., Chen, C., Chiang, E., Chilcote, J. K., Dawson, R. I., Dong, R., Doyon, R., Draper, Z. H., Duchêne, 
G., Esposito, T. M., Fabrycky, D., Fitzgerald, M. P., Follette, K. B., Fortney, J. J., Gerard, B., Goodsell, S., Greenbaum, A. Z., Hibon, P., Hinkley, S., Cotten, T. H., Hung, L. W., Ingraham, P., Johnson-Groh, M., Kalas, P., Lafreniere, D., Larkin, J. E., Lee, J., Line, M., Long, D., Maire, J., Marchis, F., Matthews, B. C., Max, C. E., Metchev, S., Millar-Blanchaer, M. A., Mittal, T., Morley, C. V., Morzinski, K. M., Murray-Clay, R., Oppenheimer, R., Palmer, D. W., Patel, R., Perrin, M. D., Poyneer, L. A., Rafikov, R. R., Rantakyrö, F. T., Rice, E. L., Rojo, P., Rudy, A. R., Ruffio, J. B., Ruiz, M. T., Sadakuni, N., Saddlemyer, L., Salama, M., Savransky, D., Schneider, A. C., Sivaramakrishnan, A., Song, I., Soummer, R., Thomas, S., Vasisht, G., Wallace, J. K., Ward- Duong, K., Wiktorowicz, S. J., Wolff, S. G., and Zuckerman, B., "Discovery and spectroscopy of the young jovian planet 51 Eri b with the Gemini Planet Imager," Science 350, 64-67 (Oct. 2015).

[9] Chauvin, G., Desidera, S., Lagrange, A. M., Vigan, A., Gratton, R., Langlois, M., Bonnefoy, M., Beuzit, J. L., Feldt, M., Mouillet, D., Meyer, M., Cheetham, A., Biller, B., Boccaletti, A., D'Orazi, V., Galicher, R., Hagelberg, J., Maire, A. L., Mesa, D., Olofsson, J., Samland, M., Schmidt, T. O. B., Sissa, E., Bonavita, M., Charnay, B., Cudel, M., Daemgen, S., Delorme, P., Janin-Potiron, P., Janson, M., Keppler, M., Le Coroller, H., Ligi, R., Marleau, G. D., Messina, S., Mollière, P., Mordasini, C., Müller, A., Peretti, S., Perrot, C., Rodet, L., Rouan, D., Zurlo, A., Dominik, C., Henning, T., Menard, F., Schmid, H. M., Turatto, M., Udry, S., Vakili, F., Abe, L., Antichi, J., Baruffolo, A., Baudoz, P., Baudrand, J., Blanchard, P., Bazzon, A., Buey, T., Carbillet, M., Carle, M., Charton, J., Cascone, E., Claudi, R., Costille, A., Deboulbe, A., De Caprio, V., Dohlen, K., Fantinel, D., Feautrier, P., Fusco, T., Gigan, P., Giro, E., Gisler, D., Gluck, L., Hubin, N., Hugot, E., Jaquet, M., Kasper, M., Madec, F., Magnard, Y., Martinez, P., Maurel, D., Le Mignant, D., Möller-Nilsson, O., Llored, M., Moulin, T., Origné, A., Pavlov, A., Perret, D., Petit, C., Pragt, J., Puget, P., Rabou, P., Ramos, J., Rigal, R., Rochat, S., Roelfsema, R., Rousset, G., Roux, A., Salasnich, B., Sauvage, J. F., Sevin, A., Soenke, C., Stadler, E., Suarez, M., Weber, L., Wildi, F., Antoniucci, S., Augereau, J. C., Baudino, J. L., Brandner, W., Engler, N., Girard, J., Gry, C., Kral, Q., Kopytova, T., Lagadec, E., Milli, J., Moutou, C., Schlieder, J., Szulágyi, J., Thalmann, C., and Wahhaj, Z., "Discovery of a warm, dusty giant planet around HIP 65426," Astronomy $\mathcal{E}$ Astrophysics 605, L9 (Sept. 2017).

[10] Kalas, P., Graham, J. R., and Clampin, M., "A planetary system as the origin of structure in Fomalhaut's dust belt," Nature 435, 1067-1070 (June 2005).

[11] Schneider, G., Debes, J. H., Grady, C. A., Gáspár, A., Henning, T., Hines, D. C., Kuchner, M. J., Perrin, M., and Wisniewski, J. P., "The HR 4796A Debris System: Discovery of Extensive Exo-ring Dust Material," Astronomical Journal 155, 77 (Feb. 2018).

[12] Spergel, D., Gehrels, N., Baltay, C., Bennett, D., Breckinridge, J., Donahue, M., Dressler, A., Gaudi, B. S., Greene, T., Guyon, O., Hirata, C., Kalirai, J., Kasdin, N. J., Macintosh, B., Moos, W., Perlmutter, S., Postman, M., Rauscher, B., Rhodes, J., Wang, Y., Weinberg, D., Benford, D., Hudson, M., Jeong, W. S., Mellier, Y., Traub, W., Yamada, T., Capak, P., Colbert, J., Masters, D., Penny, M., Savransky, D., Stern, D., Zimmerman, N., Barry, R., Bartusek, L., Carpenter, K., Cheng, E., Content, D., Dekens, F., Demers, R., Grady, K., Jackson, C., Kuan, G., Kruk, J., Melton, M., Nemati, B., Parvin, B., Poberezhskiy, I., Peddie, C., Ruffa, J., Wallace, J. K., Whipple, A., Wollack, E., and Zhao, F., "Wide-Field InfrarRed Survey Telescope-Astrophysics Focused Telescope Assets WFIRST-AFTA 2015 Report," arXiv e-prints , arXiv:1503.03757 (Mar. 2015).

[13] Mennesson, B., Gaudi, S., Seager, S., Cahoy, K., Domagal-Goldman, S., Feinberg, L., Guyon, O., Kasdin, J., Marois, C., Mawet, D., Tamura, M., Mouillet, D., Prusti, T., Quirrenbach, A., Robinson, T., Rogers, L., Scowen, P., Somerville, R., Stapelfeldt, K., Stern, D., Still, M., Turnbull, M., Booth, J., Kiessling, A., Kuan, G., and Warfield, K., "The Habitable Exoplanet (HabEx) Imaging Mission: preliminary science drivers and technical requirements," in [Space Telescopes and Instrumentation 2016: Optical, Infrared, and Millimeter Wave], MacEwen, H. A., Fazio, G. G., Lystrup, M., Batalha, N., Siegler, N., and Tong, E. C., eds., Society of Photo-Optical Instrumentation Engineers (SPIE) Conference Series 9904, 99040L (July 2016).

[14] The LUVOIR Team, "The LUVOIR Mission Concept Study Final Report," arXiv e-prints , arXiv:1912.06219 (Dec. 2019).

[15] Guyon, O., Mazin, B., Fitzgerald, M., Mawet, D., Marois, C., Skemer, A., Lozi, J., and Males, J., "Wavefront control architecture and expected performance for the TMT Planetary Systems Imager," in [Adaptive Optics 
Systems VI], Society of Photo-Optical Instrumentation Engineers (SPIE) Conference Series 10703, 107030Z (July 2018).

[16] Guyon, O., "Extreme Adaptive Optics," Annual Reviews of Astronomy \&3 Astrophysics 56, 315-355 (Sept. 2018).

[17] Beuzit, J. L., Vigan, A., Mouillet, D., Dohlen, K., Gratton, R., Boccaletti, A., Sauvage, J. F., Schmid, H. M., Langlois, M., Petit, C., Baruffolo, A., Feldt, M., Milli, J., Wahhaj, Z., Abe, L., Anselmi, U., Antichi, J., Barette, R., Baudrand, J., Baudoz, P., Bazzon, A., Bernardi, P., Blanchard, P., Brast, R., Bruno, P., Buey, T., Carbillet, M., Carle, M., Cascone, E., Chapron, F., Chauvin, G., Charton, J., Claudi, R., Costille, A., De Caprio, V., Delboulbé, A., Desidera, S., Dominik, C., Downing, M., Dupuis, O., Fabron, C., Fantinel, D., Farisato, G., Feautrier, P., Fedrigo, E., Fusco, T., Gigan, P., Ginski, C., Girard, J., Giro, E., Gisler, D., Gluck, L., Gry, C., Henning, T., Hubin, N., Hugot, E., Incorvaia, S., Jaquet, M., Kasper, M., Lagadec, E., Lagrange, A. M., Le Coroller, H., Le Mignant, D., Le Ruyet, B., Lessio, G., Lizon, J. L., Llored, M., Lundin, L., Madec, F., Magnard, Y., Marteaud, M., Martinez, P., Maurel, D., Ménard, F., Mesa, D., Möller-Nilsson, O., Moulin, T., Moutou, C., Origné, A., Parisot, J., Pavlov, A., Perret, D., Pragt, J., Puget, P., Rabou, P., Ramos, J., Reess, J. M., Rigal, F., Rochat, S., Roelfsema, R., Rousset, G., Roux, A., Saisse, M., Salasnich, B., Santambrogio, E., Scuderi, S., Segransan, D., Sevin, A., Siebenmorgen, R., Soenke, C., Stadler, E., Suarez, M., Tiphène, D., Turatto, M., Udry, S., Vakili, F., Waters, L. B. F. M., Weber, L., Wildi, F., Zins, G., and Zurlo, A., "SPHERE: the exoplanet imager for the Very Large Telescope," arXiv e-prints , arXiv:1902.04080 (Feb 2019).

[18] Lafrenière, D., Marois, C., Doyon, R., Nadeau, D., and Artigau, É., "A New Algorithm for Point-Spread Function Subtraction in High-Contrast Imaging: A Demonstration with Angular Differential Imaging," Astrophysical Journal 660, 770-780 (May 2007).

[19] Soummer, R., Pueyo, L., and Larkin, J., "Detection and Characterization of Exoplanets and Disks Using Projections on Karhunen-Loève Eigenimages," Astrophysical Journal Letters 755, L28 (Aug. 2012).

[20] Marois, C., Lafrenière, D., Doyon, R., Macintosh, B., and Nadeau, D., "Angular Differential Imaging: A Powerful High-Contrast Imaging Technique," Astrophysical Journal 641, 556-564 (Apr 2006).

[21] Sparks, W. B. and Ford, H. C., "Imaging Spectroscopy for Extrasolar Planet Detection," Astrophysical Journal 578, 543-564 (Oct. 2002).

[22] Bailey, V. P., Poyneer, L. A., Macintosh, B. A., Savransky, D., Wang, J. J., De Rosa, R. J., Follette, K. B., Ammons, S. M., Hayward, T., Ingraham, P., Maire, J., Palmer, D. W., Perrin, M. D., Rajan, A., Rantakyrö, F. T., Thomas, S., and Véran, J.-P., "Status and performance of the Gemini Planet Imager adaptive optics system," in [Adaptive Optics Systems V], Marchetti, E., Close, L. M., and Véran, J.-P., eds., Society of Photo-Optical Instrumentation Engineers (SPIE) Conference Series 9909, 99090V (July 2016).

[23] Groff, T. D., Chilcote, J., Kasdin, N. J., Galvin, M., Loomis, C., Carr, M. A., Brand t, T., Knapp, G., Limbach, M. A., Guyon, O., Jovanovic, N., McElwain, M. W., Takato, N., and Hayashi, M., "Laboratory testing and performance verification of the CHARIS integral field spectrograph," in [Ground-based and Airborne Instrumentation for Astronomy VI], Society of Photo-Optical Instrumentation Engineers (SPIE) Conference Series 9908, 990800 (Aug 2016).

[24] Currie, T., Guyon, O., Lozi, J., Groff, T., Kasdin, N. J., Martinache, F., Brandt, T. D., Chilcote, J., Marois, C., Gerard, B., Jovanovic, N., and Vievard, S., "Performance and early science with the Subaru Coronagraphic Extreme Adaptive Optics project," in [Society of Photo-Optical Instrumentation Engineers (SPIE) Conference Series], Society of Photo-Optical Instrumentation Engineers (SPIE) Conference Series 11117, 111170X (Sept. 2019).

[25] Goebel, S., Currie, T., Guyon, O., Brand t, T. D., Groff, T. D., Jovanovic, N., Kasdin, N. J., Lozi, J., Hodapp, K., Martinache, F., Grady, C., Hayashi, M., Kwon, J., McElwain, M. W., Yang, Y., and Tamura, M., "SCExAO/CHARIS Near-IR High-contrast Imaging and Integral Field Spectroscopy of the HIP 79977 Debris Disk," Astronomical Journal 156, 279 (Dec 2018).

[26] Currie, T., Brandt, T. D., Uyama, T., Nielsen, E. L., Blunt, S., Guyon, O., Tamura, M., Marois, C., Mede, K., Kuzuhara, M., Groff, T. D., Jovanovic, N., Kasdin, N. J., Lozi, J., Hodapp, K., Chilcote, J., Carson, J., Martinache, F., Goebel, S., Grady, C., McElwain, M., Akiyama, E., Asensio-Torres, R., Hayashi, M., Janson, M., Knapp, G. R., Kwon, J., Nishikawa, J., Oh, D., Schlieder, J., Serabyn, E., Sitko, M., and Skaf, 
N., "SCExAO/CHARIS Near-infrared Direct Imaging, Spectroscopy, and Forward-Modeling of $\kappa$ And b: A Likely Young, Low-gravity Superjovian Companion," Astronomical Journal 156, 291 (Dec 2018).

[27] Currie, T., Marois, C., Cieza, L., Mulders, G. D., Lawson, K., Caceres, C., Rodriguez-Ruiz, D., Wisniewski, J., Guyon, O., Brandt, T. D., Kasdin, N. J., Groff, T. D., Lozi, J., Chilcote, J., Hodapp, K., Jovanovic, N., Martinache, F., Skaf, N., Lyra, W., Tamura, M., Asensio-Torres, R., Dong, R., Grady, C., Gerard, B., Fukagawa, M., Hand, D., Hayashi, M., Henning, T., Kudo, T., Kuzuhara, M., Kwon, J., McElwain, M. W., and Uyama, T., "No Clear, Direct Evidence for Multiple Protoplanets Orbiting LkCa 15: LkCa 15 bcd are Likely Inner Disk Signals," Astrophysical Journal Letters 877, L3 (May 2019).

[28] Currie, T., Brandt, T. D., Kuzuhara, M., Chilcote, J., Guyon, O., Marois, C., Groff, T., Lozi, J., Vievard, S., Sahoo, A., Deo, V., Jovanovic, N., Martinache, F., Wagner, K., Dupuy, T. J., Wahl, M., Letawsky, M., Li, Y., Zeng, Y., Brandt, G. M., Michalik, D., Grady, C., Janson, M., Knapp, G. R., Kwon, J., Lawson, K. D., McElwain, M., Uyama, T., Wisniewski, J. P., and Tamura, M., "SCExAO/CHARIS Direct Imaging Discovery of a 20 au Separation, Low-Mass Ratio Brown Dwarf Companion to an Accelerating Sun-like Star," arXiv e-prints , arXiv:2011.08855 (Nov. 2020).

[29] Lawson, K., Currie, T., Wisniewski, J. P., Tamura, M., Schneider, G., Augereau, J.-C., Brandt, T. D., Guyon, O., Kasdin, N. J., Groff, T. D., Lozi, J., Chilcote, J., Hodapp, K., Jovanovic, N., Martinache, F., Skaf, N., Akiyama, E., Henning, T., Knapp, G. R., Kwon, J., Mayama, S., McElwain, M. W., Sitko, M. L., Asensio-Torres, R., Uyama, T., and Wagner, K., "SCExAO/CHARIS Near-infrared Integral Field Spectroscopy of the HD 15115 Debris Disk," Astronomical Journal 160, 163 (Oct. 2020).

[30] Uyama, T., Currie, T., Christiaens, V., Bae, J., Muto, T., Takahashi, S. Z., Tazaki, R., Ygouf, M., Kasdin, J. N., Groff, T., Brandt, T. D., Chilcote, J., Hayashi, M., McElwain, M. W., Guyon, O., Lozi, J., Jovanovic, N., Martinache, F., Kudo, T., Tamura, M., Akiyama, E., Beichman, C. A., Grady, C. A., Knapp, G. R., Kwon, J., Sitko, M., Takami, M., Wagner, K. R., Wisniewski, J. P., and Yang, Y., "SCExAO/CHARIS Highcontrast Imaging of Spirals and Darkening Features in the HD 34700 A Protoplanetary Disk," Astrophysical Journal 900, 135 (Sept. 2020).

[31] Norris, B. R. M., Tuthill, P., Jovanovic, N., Lozi, J., Guyon, O., Cvetojevic, N., and Martinache, F., "Diffraction-limited polarimetric imaging of protoplanetary disks and mass-loss shells with VAMPIRES," in [Advances in Optical Astronomical Instrumentation 2019], Society of Photo-Optical Instrumentation Engineers (SPIE) Conference Series 11203, 112030S (Jan. 2020).

[32] Uyama, T., Norris, B., Jovanovic, N., Lozi, J., Tuthill, P., Guyon, O., Kudo, T., Hashimoto, J., Tamura, M., and Martinache, F., "High-contrast $\mathrm{H} \alpha$ imaging with Subaru/SCExAO+VAMPIRES," arXiv e-prints , arXiv:2008.10780 (Aug. 2020).

[33] Vievard, S., Huby, E., Cvetojevic, N., Lacour, S., Martin, G., Guyon, O., Lozi, J., Kotani, T., Jovanovic, N., Perrin, G. S., Marchis, F., Duchêne, G., Lapeyrère, V., Rouan, D., and Barjot, K., "FIRST, a PupilRemapping Fiber Interferometer at the Subaru Telescope," Society of Photo-Optical Instrumentation Engineers (SPIE) Conference Series 11446, 1144654 (2020).

[34] Martinod, M. A., Norris, B., Gross, S., Arriola, A., Gretzinger, T., Withford, M., Lagadec, T., and Tuthill, P., "Imaging exoplanets with nulling interferometry using integrated-photonics: the GLINT project," in [Advances in Optical Astronomical Instrumentation 2019], Society of Photo-Optical Instrumentation Engineers (SPIE) Conference Series 11203, 112030R (Jan. 2020).

[35] Walter, A. B., Fruitwala, N., Steiger, S., Bailey, John I., I., Zobrist, N., Swimmer, N., Lipartito, I., Smith, J. P., Meeker, S. R., Bockstiegel, C., Coiffard, G., Dodkins, R., Szypryt, P., Davis, K. K., Daal, M., Bumble, B., Collura, G., Guyon, O., Lozi, J., Vievard, S., Jovanovic, N., Martinache, F., Currie, T., and Mazin, B. A., "The MKID Exoplanet Camera for Subaru SCExAO," Publications of the Astronomical Society of the Pacific 132, 125005 (Dec. 2020).

[36] Kotani, T., Kawahara, H., Ishizuka, M., Jovanovic, N., Guyon, Olivier amd Vievard, S., Lozi, J., Sahoo, A., Yoneta, K., and Tamura, M., "The REACH project: Combining extremely high-contrast and high spectral resolution at the Subaru Telescope," Society of Photo-Optical Instrumentation Engineers (SPIE) Conference Series 11448, 11448-299 (2020).

[37] Lozi, J., Guyon, O., Kudo, T., Zhang, J., Jovanovic, N., Norris, B., Martinod, M.-A., Groff, T. D. G., Chilcote, J., Tamura, M., Bos, S., Snik, F., Vievard, S., Sahoo, A. S., Martinache, F., and Kasdin, J., "New 
NIR spectro-polarimetric modes for the SCExAO instrument," Society of Photo-Optical Instrumentation Engineers (SPIE) Conference Series 11448, 11448-304 (2020).

[38] Rains, A. D., Ireland, M. J., Jovanovic, N., Bento, J., Feger, T., Lozi, J., Schwab, C., Coutts, D. W., Guyon, O., Arriola, A., Gross, S., and Harris, J. E., "Development of the single-mode fiber integral field unit for the RHEA Spectrograph," in [Ground-based and Airborne Instrumentation for Astronomy VII], Evans, C. J., Simard, L., and Takami, H., eds., Society of Photo-Optical Instrumentation Engineers (SPIE) Conference Series 10702, 107025J (July 2018).

[39] Guyon, O., Sevin, A., Gratadour, D., Bernard, J., Ltaief, H., Sukkari, D., Cetre, S., Skaf, N., Lozi, J., Martinache, F., Clergeon, C., Norris, B., Wong, A., and Males, J., "The compute and control for adaptive optics (CACAO) real-time control software package," in [Adaptive Optics Systems VI], Close, L. M., Schreiber, L., and Schmidt, D., eds., Society of Photo-Optical Instrumentation Engineers (SPIE) Conference Series 10703, 107031E (July 2018).

[40] Miller, K., Guyon, O., and Males, J., "Spatial linear dark field control: stabilizing deep contrast for exoplanet imaging using bright speckles," Journal of Astronomical Telescopes, Instruments, and Systems 3, 049002 (Oct 2017).

[41] Currie, T., Pluzhnik, E., Guyon, O., Belikov, R., Miller, K., Bos, S., Males, J., Sirbu, D., Bond, C., Frazin, R., Groff, T., Kern, B., Lozi, J., Mazin, B. A., Nemati, B., Norris, B., Subedi, H., and Will, S., "Laboratory Demonstration of Spatial Linear Dark Field Control For Imaging Extrasolar Planets in Reflected Light," Publications of the Astronomical Society of the Pacific 132, 104502 (Oct. 2020).

[42] Bendek, E., Sirbu, D., Belikov, R., Lozi, J., Guyon, O., Pluzhnik, E., and Currie, T., "Demonstration of multi-star wavefront control using SCExAO," in [Techniques and Instrumentation for Detection of Exoplanets IX], Shaklan, S. B., ed., 11117, $301-311$, International Society for Optics and Photonics, SPIE (2019).

[43] Guyon, O., "Limits of Adaptive Optics for High-Contrast Imaging," Astrophysical Journal 629, 592-614 (Aug. 2005). 